\title{
Retinal Biometric System using Electromagnetism-like Optimization Algorithm
}

\author{
Sivakamasundari. J, Jesintha Rani. D, Mohanapriya. S, Raksha. G
}

\begin{abstract}
Biometric system is the technology used for the purpose of identifying the physiological and behavioural characteristics of an individual as input, analyzes it and identifies the individual as a genuine or imposter. Among all biometrics, retina based identification is perceived as a robust, unforgeable and reliable form of biometric solution. The blood vasculatures of retina are unique and used as features for retinal biometric system. In this work, an attempt has been made to employ an Electromagnetism-like Optimization Algorithm (EMOA) with Otsu Multilevel Thresholding (MLT) for segmentation of vascular pattern from the retinal fundus images for retinal biometric system. Retinal images are taken from the publicly available database such as DRIVE, STARE and HRF. The original images are subjected to preprocessing. Segmentation is carried out on the preprocessed images using EMOA Based Otsu MLT. This method provides comparatively better segmentation accuracy of 0.974 than other existing methods. Texture and vessel features are extracted from the segmented image. Matching is done between query and enrolled images using Euclidian distance measure. Decision is made using best matched image. This biometric system shows matching accuracy of $97 \%$. Hence, this method could be recommended for retinal biometric system.
\end{abstract}

Index Terms: Biometric system, vasculature, segmentation, electromagnetism-like optimization, multilevel thresholding, retinal fundus image, matching

\section{INTRODUCTION}

Biometrics is a method for recognizing a person's physiological or behavioural characteristics. It describe about body measurement and calculations [1]. It is categorized as physiological and behavioural biometrics. Physiological biometrics is the physical character measured for identification and verification which includes face recognition, fingerprint, hand geometry, iris recognition and DNA. Behavioural biometric is characters of human behaviour. It includes include keystroke, signature and voice recognition [2].

A biometric system provides assurance of authentication, easy to use and the end user does not need to remember complex passwords. The application of biometrics based authentication include workstation, network, domain access, security purposes, transaction security for banking, web security and healthcare industry [3].

Revised Manuscript Received on July 22, 2019

J. Sivakamasundari, Department of Biomedical Engineering, Jerusalem College of Engineering, Chennai, India.

D. Jesintha Rani, Department of Biomedical Engineering, Jerusalem College of Engineering, Chennai, India.

S. Mohanapriya, Department of Biomedical Engineering, Jerusalem

G. Raksha, Department of Biomedical Engineering, Jerusalem College of Engineering, Chennai, India. College of Engineering, Chennai, India.

A person's retinal vasculature is used in the biometric system that identifies a person using unique patterns of the retina. The blood vasculatures in the retina are not entirely same even in identical twins. The vessel pattern of retina is altered due to retinal degenerative disorders such as diabetic retinopathy and glaucoma. Otherwise, it remains unchanged from birth until death. Due to its unique and unchanging nature, low occurrence of false positives, extremely low false negative rates, retina appears to be the most precise, speedy results and reliable biometric [4].

Segmentation is an important scheme in the retinal biometric system. The retinal images consist of thin vessels in low gray intensities than wide vessels and this is a challenging task for segmentation. Various vessel segmentation algorithms such as vessel tracking, filter based, classifier and morphological based methods, pixel classification, line cross-sections with edge enhancement, modified matched filtering and continuation algorithm have been employed [ 5 , $6,7]$.

Thresholding is a significant technique used in many region based segmentation. An image is segmented into two classes by one threshold point in bi-level thresholding, whereas in multilevel thresholding the image pixels are divided into several classes. The non-parametric bi-level threshold selection techniques such as Otsu and Kapur methods are used for image segmentation and are extended to multilevel thresholding $[8,9,10]$.

In this work, a retinal biometric system is developed. Initially, retinal fundus images are subjected to preprocessing steps such as green channel and morphological operations. Then, EMOA based Otsu MLT is employed on the preprocessed images to segment blood vessels. The validation of segmentation is carried out with its ground truth images. Statistical and texture features are obtained from the segmented vasculatures. At last, matching accuracy is tested on the query image with enrolled images using Euclidean Distance measure.

\section{METHODOLOGY}

\section{A. Retinal Image Database}

Original 50 digital retinal fundus images are considered for this work. These images are taken from the publicly available database such as Digital Retinal Images for Vessel Extraction (DRIVE), Structured Analysis of the Retina (STARE) and High Resolution Fundus (HRF) [11]. These images are used for testing and training of retinal biometric system.

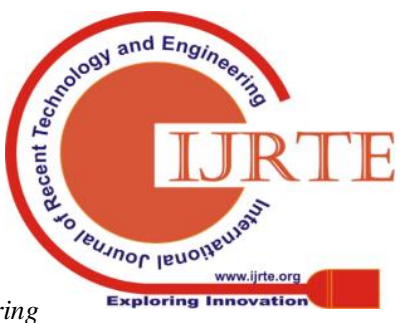
\& Sciences Publication 


\section{B. Preprocessing Steps}

The retinal images of different sizes are resized into $500 \mathrm{x}$ 500 . The green part of image produces the clear vessels and contrast among the optic disc and tissue from background. The green channel images are treated by morphological filters such as top-hat and bottom-hat to improve the blood vessel contrast and back ground is removed using subtracting operation [12].

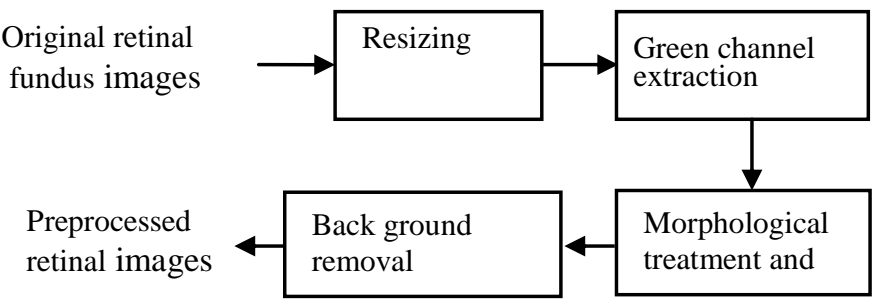

Fig. 1. Preprocessing steps

\section{Segmentation}

The Otsu's criterion selects optimal threshold points by maximizing the variance between classes. In multilevel thresholding (MLT) based segmentation, selection of optimal threshold points increases the computational complexity when a new threshold level is introduced. Evolutionary Optimization (EO) based thresholding methods handles this problem to select better threshold points than the classical techniques. The EMOA combined with the Otsu MLT algorithm which is efficiently identify the optimal threshold points to segment the different regions of general images [13].

Thresholding is a process where the pixels of a gray scale image are divided into sets or classes depending on their intensity level $(L)$ [8]. In bi-level thresholding, pixels of the image are partitioned into two classes such as $C_{1}$ with gray levels $(0 \ldots, t h)$ and $C_{2}$ with gray levels $(t h . \ldots, L-1)$. The selection of threshold value $(t h)$ for two different classes is given as:

$$
\begin{array}{ll}
C_{1} \leftarrow p \text { if } & 0 \leq p<t h \\
C_{2} \leftarrow p \text { if } & t h \leq p<L-1
\end{array}
$$

where $p$ is one of the $\mathrm{m} \times \mathrm{n}$ pixels of the gray scale image $I_{g}$ and it is represented in $L$ gray scale levels $L=\{0,1,2, \ldots$. $L-1\}$. A global threshold value is selected by maximizing the separable classes in gray levels [9]. The specific intensity of pixel $i$ at gray level $(0 \leq i \leq L-1)$ is denoted by $n_{i}$. It is the frequency of occurrence in the total number of pixels $N=$ $n_{1}+n_{2}+\ldots \ldots+n_{L}$, where $L$ represents the gray level intensity values in the histogram. Then, the histogram is normalized in a probability of occurrence with gray level $i$ and is given as:

$$
P_{i}^{c}=\frac{n_{i}^{c}}{N}, \sum_{i=1}^{N} P_{i}^{c}=1
$$

where the image component $(c)$ is one for gray scale images. The optimal threshold or the objective function, $J(t)$ for bi-level thresholding is obtained by maximizing the between class variance $\sigma_{B C}^{2}$ and is given as:

$$
f(t h)=\underset{0 \leq t h \leq L-1}{\arg \max }\left\{\sigma_{B C}^{2}(t h)\right\}
$$

Equation (3) is extended for multiple classes of $k$ as follows:

$$
\begin{array}{ll}
C_{1} \leftarrow p \text { if } & 0 \leq p<t h_{1} \\
C_{2} \leftarrow p \text { if } & t h_{1} \leq p<t h_{2} \\
C_{i} \leftarrow p \text { if } & t h_{i} \leq p<t h_{i+1} \\
C_{k} \leftarrow p \text { if } & t h_{k} \leq p<L-1
\end{array}
$$

where $\left\{t h_{1}, t h_{2}, \ldots \ldots . t h_{i}, t h_{i+1}, t h_{k}\right\}$ represents the different thresholds. The objective function is $J(T H)$ where $\boldsymbol{T H}=\left\{t \boldsymbol{h}_{1}, t \boldsymbol{h}_{2}, \ldots, t_{k-1}\right\}$ is a vector containing multiple thresholds obtained by maximizing the variances $\sigma_{B C}^{2}$. The objective function is derived as:

$$
\begin{aligned}
J(T H)= & \left(t h_{1}, t h_{2}, \ldots, t_{k-1}\right) \\
& =\max \left\{\sigma_{B C}^{2}\left(t h_{1}, t h_{2}, \ldots, t_{k-1}\right)\right\}, \\
0 & \leq t h_{i} \leq L-1, \quad i=1,2, \ldots . ., k
\end{aligned}
$$

where $i$ represents the specific class. The between-class variance $\sigma_{B C}^{2}$ is used to measure the separation among all classes of $k$ [10].

\section{Multilevel Thresholding by EMOA}

The Electromagnetism like optimization algorithm mimics the attraction-repulsion mechanism among charges to move the members of a population towards an optimal solution. According to the electromagnetism theory, a particle is treated as a solution and has a charge. The charge of each point $x_{i, t} \in S_{t}$ in the search space $\mathbf{X}$ relates to an objective function to be optimized. A better solution contains a stronger charge than other points. In the attraction-repulsion mechanism process of EMO, the points with more charge attract other points in $\mathrm{St}$, and the points with less charge repel other points. At last, a total force vector $F_{i}{ }^{t}$ is calculated by the force exerted in the $\mathrm{i}^{\text {th }}$ point of particle $x_{i, t} \in S_{t}$ and adding these attraction and repulsion forces that moved in the direction to a location $\mathrm{y}_{\mathrm{i}, \mathrm{t}}$, where $\mathrm{St}$ represents the population set, $x_{i, t}$ and $y_{i, t}$ are each point of charged particles in the set. The objective function values $\mathrm{f}\left(\mathrm{x}_{\mathrm{i}, \mathrm{t}}\right)$ are computed and the best point is identifies as follows:

$$
x_{t}^{B}=\arg \max _{x_{i, t} \in S_{t}}\left\{f\left(x_{i, t}\right)\right\}
$$

where $x_{t}^{B}$ is the element of St that produces the maximum value in terms of the objective function $\mathrm{f}$. The total force $F_{i}{ }^{t}$, corresponding to $\mathrm{xi}, \mathrm{t}$ is calculates as:

$$
F_{i}^{t}=\sum_{j=1, i \neq 1}^{N} F_{i, j}^{t}
$$

The point $\mathrm{x}_{\mathrm{i}, \mathrm{t}}$ is moved along the total $F_{i}^{t}$ except for $x_{t}^{B}$ as:

$$
x_{i, t}=x_{i, t}+\lambda \frac{F_{i}^{t}}{\left\|F_{i}^{t}\right\|}(R N G), \quad i=1,2, \ldots ., N ; i \neq B
$$

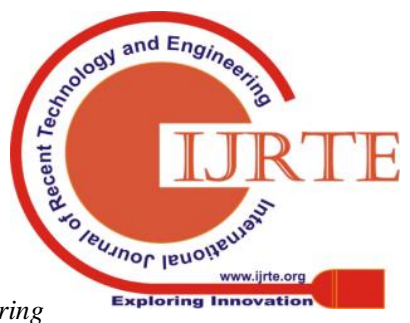


where the random step length $\lambda=$ random $(0,1)$, and $\mathrm{RNG}$ denotes the allowed feasible range of movement towards the upper bound $\mathrm{u}_{\mathrm{i}}$ or lower bound $\mathrm{l}_{\mathrm{i}}$.

EMOA exhibits significant search capabilities and generates a multilevel segmentation technique which can effectively identify the threshold points of an image in a reduced number of iterations. Each particle uses k different elements as decision variables within the optimization algorithm. Such decision variables represent a different threshold point ' $t h$ ' which is used for the segmentation. Therefore, the complete population is represented as:

$$
S_{t}=\left[T H_{1}^{c}, T H_{2}^{c}, \ldots, T H_{N}^{c}\right], \quad T H_{i}^{c}=\left[t h_{1}^{c}, t h_{2}^{c}, \ldots, t_{k}^{c}\right]^{T}
$$

where $\mathrm{t}$ represents the iteration number, $\mathrm{T}$ refers to the transpose operator, $\mathrm{N}$ is the size of population and $\mathrm{c}$ is the image component. The EMOA based Otsu MLT segmentation algorithm is implemented considering the Otsu objective function given in the Equation (5) [13]. The best objective functions or the optimal threshold points are used for the retinal image segmentation.

\section{E. Validation of Segmentation}

The quantitative validation of segmentation is carried out by comparing the segmented blood vessels with its corresponding ground truth using binary similarity measures. The similarity values close to one is the greatest matching measures [14]. The validation of segmentation is also done by comparing the accuracy of EMOA based Otsu MLT method in healthy images of DRIVE and HRF database with the other existing methods.

\section{F. Feature Extraction}

The statistical, textural and structural features are obtained from the segmented images and analyzed using statistical significance t-test. Images similar to query image are ranked and retrieval is carried out [12].

\section{G. Matching and Decision}

Matching is done with respect to $1: 1$ matching and $1: \mathrm{n}$ matching. This process is important for identification and verification process to identify whether the person is genuine or an imposter. The retrieval of retinal image is carried out using similarity matching between the features of query and database images using Euclidean Distance (ED) measures and is given by,

$$
E D_{(r, s)}=\left(\sum_{i=1}^{N}\left(r_{i}-s_{i}\right)^{2}\right)^{1 / 2}
$$

where $r_{i}$ and $s_{i}$ represent the feature vectors of database image and query image respectively and $\mathrm{N}$ is the number of elements of the descriptors. The shorter distance corresponds to higher similarity matching [12].

\section{RESULTS AND DISCUSSION}

Input parameters assigned for the EMOA for segmentation of blood vessels are given in Table I. Typical retinal image is shown in Fig. 2 (a). Green channel of the original image improves vessel contrast from its background as shown in Fig. 2 (b). The top-hat MO reduces the non-uniform illumination and bottom-hat MO improves the contrast of primary and thin vessels further as shown in Fig. 2 (c). The background removed image is shown in Fig. 2 (d). The segmentation using EMOA-based Otsu MLT for normal image and the corresponding ground truth are shown in Fig. 2 (e) and (f).

Table I Input Parameters for EMOA

\begin{tabular}{|l|l|}
\hline PARAMETERS & VALUES \\
\hline Threshold levels & 3 \\
\hline Dimensions & 3 \\
\hline Number of local search & 4 \\
\hline $\begin{array}{c}\text { Multiplier for the neighborhood } \\
\text { search }(\delta)\end{array}$ & 0.025 \\
\hline Population size & 40 \\
\hline Maximum number of iterations & 150 \\
\hline
\end{tabular}

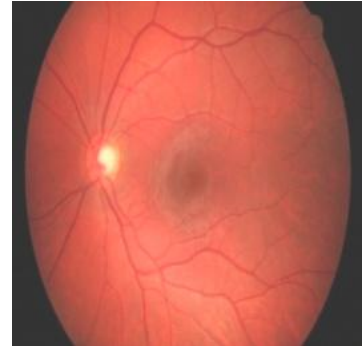

(a)

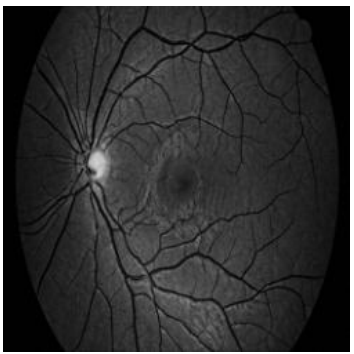

(c)

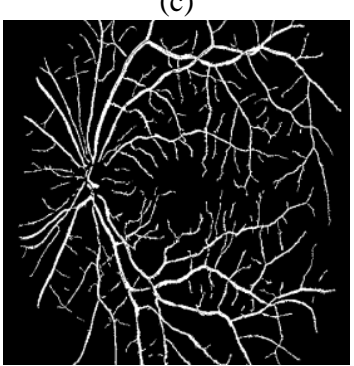

(e)

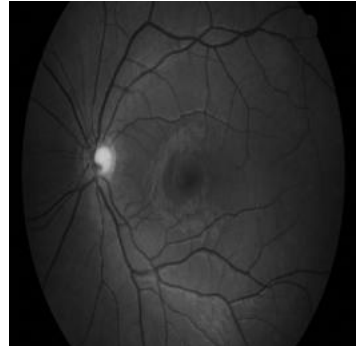

(b)

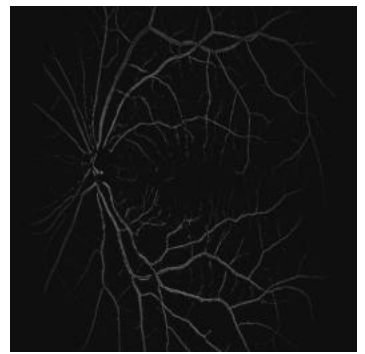

(d)

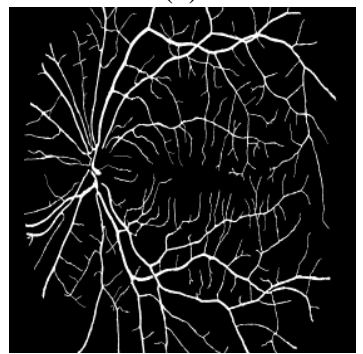

(f)
Fig. 2. Pre-processing and segmentation of normal image in HRF database (a) original normal, (b) green channel, (c) morphological pre-processing, (d) back ground removed, (e) EMOA-based Otsu MLT segmented and (f) ground truth image

This technique detects all the primary, wide and small vessels up to the entire length. It identifies most of the thin vessel pixels without discontinuities. This shows the preservation of blood vessel map and better similarity with the ground truth image. This approach provides good search capabilities and selects the best objective functions (threshold points) from Otsu's MLT algorithm.

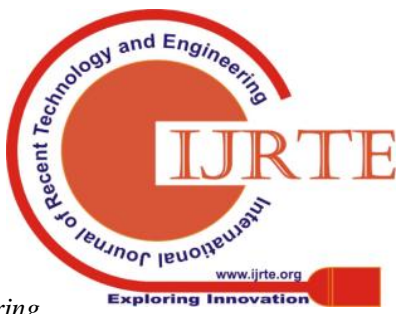


The qualitative comparison is carried out with EMOA based Otsu MLT method with different existing techniques. A normal retinal image from the DRIVE database is shown in Fig. 3 (a) and its segmentation results is shown in Fig. 3 (h) and its corresponding gold standard image is represented in Fig. 3 (i). The result of EMOA based Otsu MLT method is qualitatively better similarity with its corresponding ground truth image and comparatively better than the existing methods tested by other authors [15, 16, 17, 7, 18, 19].

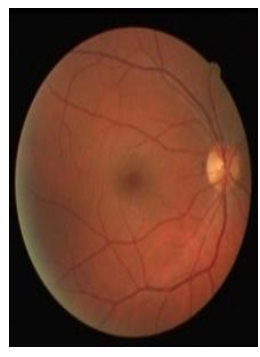

(a)

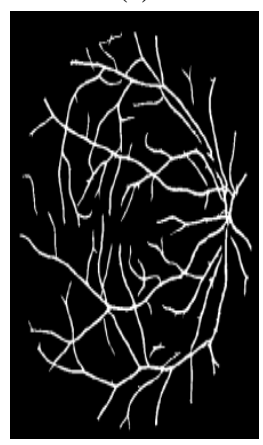

(d)

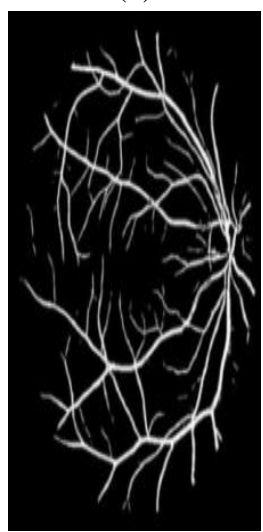

(g)

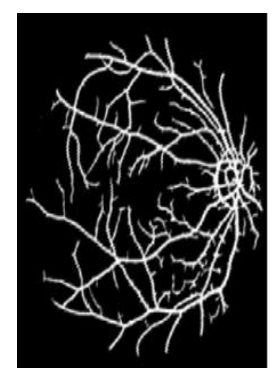

(b)

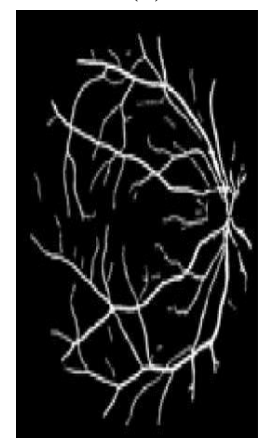

(e)

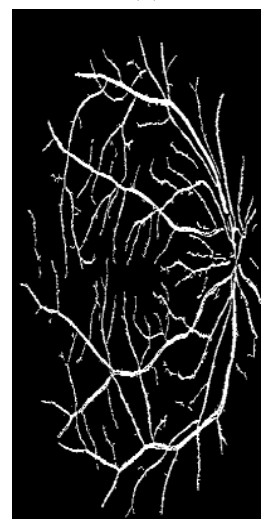

(h)

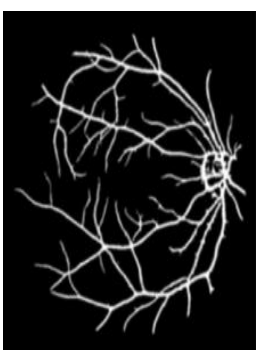

(c)

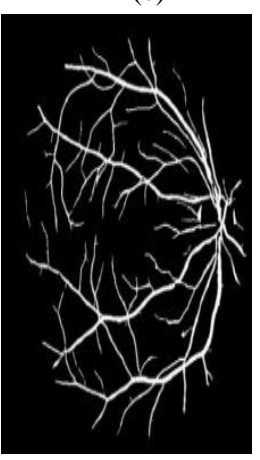

(f)

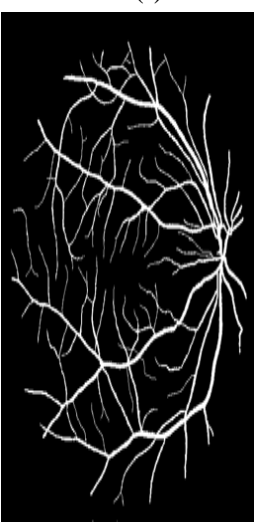

(i)
Fig. 3, Segmentation of retinal blood vessels from the DRIVE database using different methods: (a) original normal image, (b) [15], (c) [16], (d) [17], (e) [7], (f) 18, (g) [19], (h) EMOA-based Otsu MLT method and (i) ground truth image

The validation of segmentation is carried out by comparing the EMOA segmented images with corresponding ground truth images using significant binary similarity measures. The similarity values close to one are represented in Fig. 4 by means of box plots. The similarity measures such as Sokal and Michener as well as Rogers and Tanimoto shows longer whiskers and larger mean than the median indicates the skew in a positive direction. The median of Hamann measure rising towards the top of the box indicates positive skewness. The Sokal-Sneath-2 as well as Goodman and Kruskal Lambda measures achieve higher magnitudes of above 0.97 which are close to one and show quantitatively better similarity against the ground truth images.

The normalized average difference in statistical texture and vessel count are obtained from the segmented normal images and are given in Table II. Average difference in all the features are found to be highly statistically significant $(\mathrm{p}<0.0001)$.

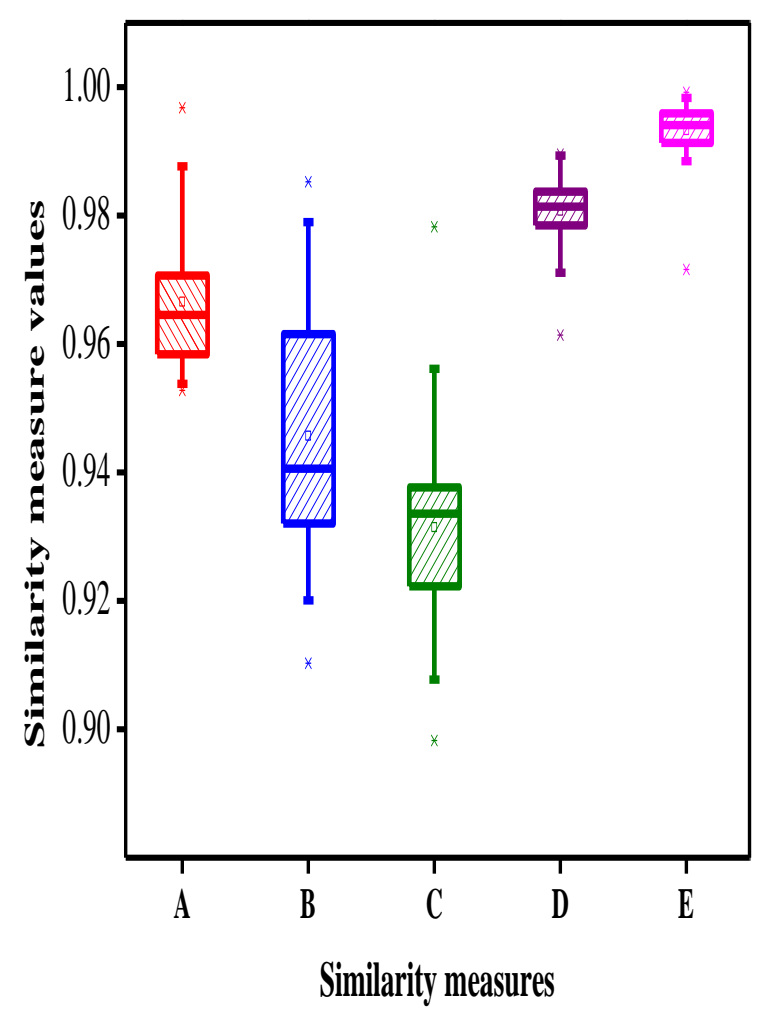

Fig. 4. Variation of binary similarity measures (A: Sokal and Michener, B: Rogers and Tanimoto; C: Hamann; D: Sokal-Sneath-2; E: Goodman and Kruskal Lambda).

TABLE I. AVERAGE VALUES OF FEATURES

\begin{tabular}{|c|c|c|}
\hline Feature Name & $\begin{array}{c}\text { Normal } \\
(\text { Mean } \pm \text { SD })\end{array}$ & $p$-values \\
\hline Energy & $0.42 \pm 0.01$ & 0.0001 \\
\hline Entropy & $0.67 \pm 0.06$ & 0.0001 \\
\hline Contrast & $0.42 \pm 0.09$ & 0.0001 \\
\hline Homogeneity & $0.84 \pm 0.03$ & 0.0001 \\
\hline $\begin{array}{l}\text { Maximum } \\
\text { probability }\end{array}$ & $0.72 \pm 0.01$ & 0.0001 \\
\hline Standard deviation & $0.62 \pm 0.06$ & 0.0001 \\
\hline Vessel count & $0.22 \pm 0.14$ & 0.0001 \\
\hline
\end{tabular}

The performance evaluation of EMOA-based Otsu MLT method is conducted on 15 healthy images and 15 DR images of the HRF database and 20 healthy images of the DRIVE database. The segmentation performance metrics is observed that the EMOA-based Otsu MLT method achieved the average segmentation accuracy of 0.974 in DRIVE database and 0.979 in HRF database.

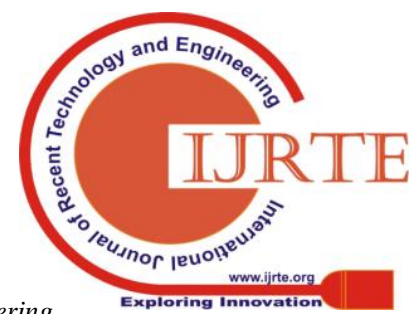


The EMOA-based Otsu MLT performance measure namely matching efficiency is $97 \%$.

\section{CONCLUSION}

The verification of physiological or behavioral features of an individual is becoming an important requirement in a variety of applications. Retinal biometric system is a significant one because it uses unique retinal vasculature pattern for each individual and almost impossible to forge. In this work, retinal images are preprocessed and vasculatures are segmented using EMOA based Otsu MLT method. Then, the validation of segmentation is carried out against the ground truth images. The performance metrics of segmentation are compared with other methods. Statistical, textural and structural features are obtained from the segmented images and analyzed. Similarity matching between query and enrolled images are carried out. From the experimental results, it is observed that the EMOA based Otsu MLT method selects the best optimized threshold points with less number of iterations. This retains wide as well as thin blood vessels in normal retinal images.

The validation of segmentation achieves higher binary similarity measure values of 0.97. This method provides a high degree of mean segmentation accuracy of 0.974 and 0.979 for normal images in the DRIVE and HRF database respectively. Statistical texture features are used for verification process to match the query image with already enrolled images. These indicate that the EMOA based biometric system shows matching accuracy of $97 \%$. Hence, this method could be used for the automatic retinal biometric system.

\section{REFERENCES}

1. I. M .Alsaadi, "Physiological biometric authentication systems, advantages disadvantages and future development: A review," Int J Sci Technol Res, vol. 12, pp. 285-289, 2015.

2. T. Sabhanayagam, V. Prasanna Venkatesan and K. Senthamaraikannan, "A Comprehensive Survey on Various Biometric Systems," Int J Applied Engg Res, vol.13, pp. 2276-2297, 2018.

3. Alfred, C. Weaver, "Biometric Authentication," IEEE Computer Society, vol.39, pp. 96-97, 2006.

4. M. Usman Akram, Anam Tariqy and A. Shoa'b Khanz, "Retinal Recognition: Personal Identification using Blood Vessels," P $6^{\text {th }}$ Int C Internet Tech and Sec Trans, pp. 180-184, 2011.

5. A. Budai, R. Bock, A. Maier, J. Hornegger and G. Michelson, G, "Robust vessel segmentation in fundus images," Int J Biomed Imag, vol. 2013, pp. 1-11, 2013.

6. J. Staal, M. D. Abràmoff, M. Niemeijer, M.A. Viergever, and B. V. Ginneken, "Ridge-based vessel segmentation in color images of the retina," IEEE Trans Med Imag, vol. 23, pp. 501-509, 2004.

7. D. Marín, A. Aquino, M. E. Gegundez-Arias and J. M. Bravo, "A new supervised method for blood vessel segmentation in retinal images by using gray-level and moment invariants-based features', IEEE Trans Med Imag, vol. 30, pp. 146-158, 2011.

8. D. Y. Huang, T. W. Lin, and W. C. Hu, "Automatic multilevel thresholding based on two-stage Otsu's method with cluster determination by valley estimation," Int. J. Innov Comput. I., vol. 7, pp. 5631-5644, 2011

9. S. Arora, J. Acharya, A. Verma, and P.K. Panigrahi, "Multilevel thresholding for image segmentation through a fast statistical recursive algorithm," Pattern Recogn. Lett., vol.29, pp. 119-125, 2008.

10.P. S. Liao, T. S. Chen, and P. C. Chung, "A fast algorithm for multilevel thresholding,” J. Inf. Sci. Eng., vol. 17, pp. 713-727, 2001.

11.T. Köhler, A. Budai, M. F. Kraus, J . Odstrcilik, G . Michelson, and J. Hornegger, "Automatic no-reference quality assessment for retinal fundus images using vessel segmentation," P IEEE 26th Int sympo comput med s, pp. 95-100, 2013

12.J. Sivakamasundari and V. Natarajan, "Design of content based image retrieval scheme for diabetic retinopathy images using harmony search algorithm,” Biomed Sci Inst, Instrument Society of America, vol. 51, pp. 273-80, 2015

13. D. Oliva, E. Cuevas, G. Pajares, D. Zaldivar and V. Osuna, "A multilevel thresholding algorithm using electromagnetism optimization," Neurocomputing, vol. 139, pp. 357-381, 2014.

14.S. S. Choi, S. H. Cha and C. Tappet, "A survey of Binary similarity and distance measures," Sys, Cyber Info, vol. 8, pp. 43-48, 2010

15.P. C. Siddalingaswamy and G. K. Prabhu, "Automatic detection of multiple oriented blood vessels in retinal images," J Biomed Sci Engg, vol. 3, pp. 101-107, 2010.

16.J. Odstrcilik, R. Kolar, A. Budai, J. Hornegger, J. Jan, J. Gazarek, T. Kubena, P. Cernosek, O. Svoboda and E. Angelopoulou, "Retinal vessel segmentation by improved matched filtering: evaluation on a new high resolution fundus image database," IET Image Processing, vol. 7, pp 373-383, 2013

17.B. Dai, W. Bu, X. Wu and Y. Zheng, "Retinal blood vessel detection using multiscale line filter and phase congruency," P Int C on imag proc, comput vision, and pat recog, pp. 235-241, 2013

18.Y. Q. Zhao, X. H. Wang, X. F. Wang and F. Y. Shih, "Retinal vessels segmentation based on level set and region growing," Pat Recog, vol. 47, pp. 2437-2446, 2014.

19.J. V. B. Soares, J. J. G. Leandro, R. M. Cesar, H. F. Jelinek and M. J. Cree, "Retinal vessel segmentation using the 2-D Gabor wavelet and supervised classification", IEEE Trans Med Imag, vol. 25, pp. 1214-1222, 2006

20.Rajesh, M., and J. M. Gnanasekar. "Path Observation Based Physical Routing Protocol for Wireless Ad Hoc Networks." Wireless Personal Communications 97.1 (2017): 1267-1289.

21.N. Nithiyanandam, K. Venkatesh, M. Rajesh, Transfer The Levels Of The Monitored Carbon, Nitrogen Gases From The Industries, International Journal of Recent Technology and Engineering, Volume-7 Issue-6S3 April, 2019.

22.Sivanesh Kumar, A., Brittoraj, S., Rajesh, M., Implementation of RFID with internet of things, Journal of Recent Technology and Engineering, Volume-7 Issue-6S3 April, 2019.

23.Rajesh, M., Sairam, R., Big data and health care system using mlearningJournal of Recent Technology and Engineering, Volume-7 Issue-6S3 April, 2019.

\section{AUTHORS PROFILE}

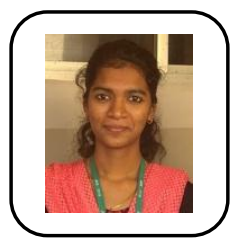

Dr. J.Sivakamasundari is working as Associate Professor in Department of Biomedical Engineering, Jerusalem college of Engineering, Chennai, India She has done B.E (ECE), M.E (AE) and Ph.D in image processing. Her research interests include biomedical image processing and data mining. She published 7 National and International journals and presented 7 International conferences.

Email: sivakamasundarij17@gmail.com

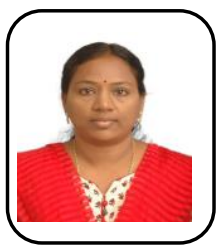

D. Jesintha Rani is studying third year Biomedical Engineering in Jerusalem college of Engineering, Chennai, India. Email: jsrani98@gmail.com

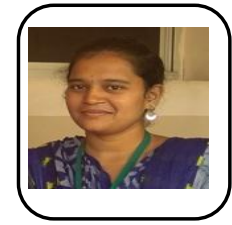

S. Mohanapriya is studying third year Biomedical Engineering in Jerusalem college of Engineering, Chennai, India. Email: priya4122549@gmail.com

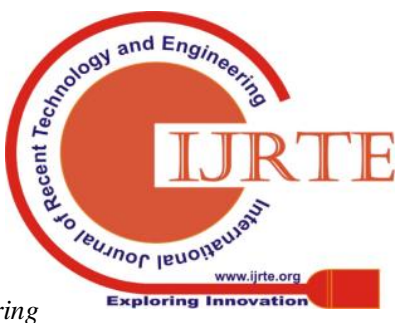


Retinal Biometric System using Electromagnetism-like Optimization Algorithm

G. Raksha is studying third year Biomedical

Engineering in Jerusalem college of Engineering,

Chennai, India. Email: rakshagrajan@gmail.com 